\title{
SELECTED CPV RESULTS FROM LHCb RUN 1 AND PROSPECTS FOR CKM $\gamma$ ANGLE MEASUREMENTS IN RUN 2*
}

\author{
AgnieszKa OBlakowska-Mucha \\ on behalf of the LHCb Collaboration \\ Faculty of Physics and Applied Computer Science \\ AGH University of Science and Technology \\ al. Mickiewicza 30, 30-059 Kraków, Poland
}

(Received April 25, 2016)

\begin{abstract}
The LHCb detector is a single-arm forward spectrometer that collects data at the LHC, designed for studies of flavour physics with high precision. In this review, a few selected results regarding $\mathrm{CP}$ violation are discussed with particular emphasis on the CKM $\gamma$ angle measurements. This summary covers results based on the data collected by the LHCb detector during 2011 and 2012 proton-proton LHC runs at the centre-of-mass energies of 7 and $8 \mathrm{TeV}$, respectively. Some remarks on prospects for analyses foreseen in the ongoing LHC Run 2 are also presented.
\end{abstract}

DOI:10.5506/APhysPolB.47.1553

\section{Introduction}

Studies of CP violation in heavy flavour physics are amongst the main interests of the LHCb Collaboration, since they can unveil indirect evidences of new physics.

Precise measurements from $\mathrm{LHCb}$ can reveal inconsistencies with respect to the theoretical predictions based on the validity of the Standard Model (SM). CP-violating effects can be searched for by over-constraining the angles of the unitary triangle of the CKM matrix (Fig. 1).

In this summary, selected results regarding $\mathrm{CP}$ violation are presented, i.e. direct searches of $\mathrm{CP}$ violation in $B$ meson decays to two and to three hadrons, measurement of $\sin 2 \beta$ from $B^{0} \rightarrow J / \psi K_{\mathrm{S}}^{0}$ and of the angle $\gamma$ from $B^{ \pm}$and $B_{s}^{0} \rightarrow D K$ decays. Results on searches of $\mathrm{CP}$ violation in charm are also discussed.

* Presented at the Cracow Epiphany Conference on the Physics in LHC Run 2, Kraków, Poland, January 7-9, 2016. 


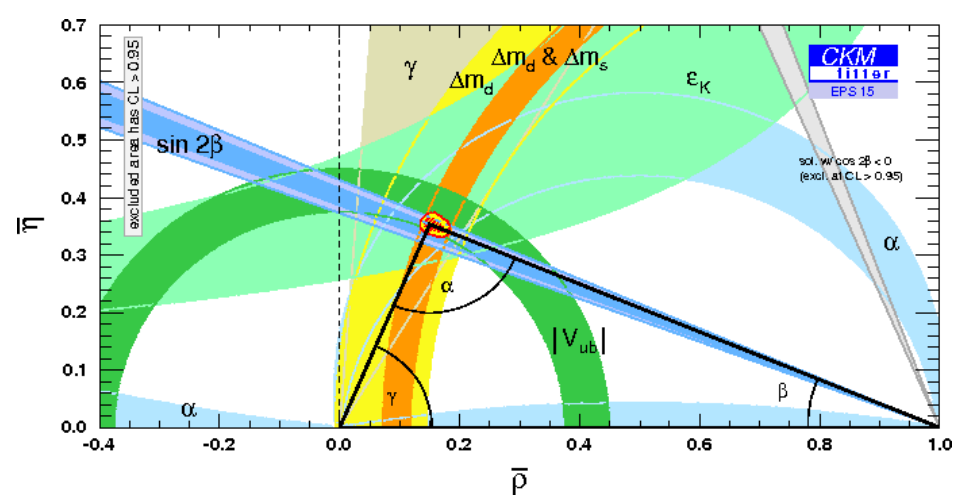

Fig. 1. The Unitary Triangle with the latest experimental values [1].

The $\mathrm{LHCb}$ experiment (Fig. 2) is a dedicated apparatus for studying flavour physics at the LHC at CERN. In particular, the experiment is designed to study $\mathrm{CP}$ violation and rare decays of beauty and charm particles. It is a single-arm forward spectrometer covering the pseudorapidity range of $2<\eta<5$. Thus, the LHCb programme is highly complementary to the direct searches performed by the ATLAS and CMS experiments.

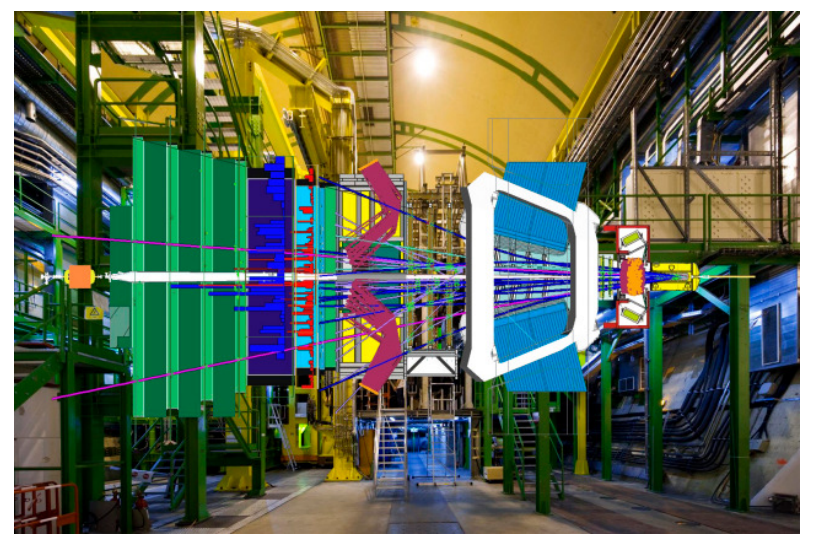

Fig. 2. The LHCb forward spectrometer at the LHC with a sample event superimposed.

The $b \bar{b}$ pair production in proton-proton collisions at the LHC is spatially correlated and occurs predominantly at small angles with respect to the beam axis. The interaction point is surrounded by the Vertex Locator. The role of this sub-detector is to precisely determine both the position of primary and secondary vertices and allowing for efficient selection of the decays of beauty and charm particles. In the Run 1 data taking, during the years 2010-2012, LHCb collected data corresponding to an integrated 
luminosity of $3 \mathrm{fb}^{-1}$. The spectrometer achieved an excellent vertex resolution, momentum determination with a precision of $\delta p / p \sim 0.4-0.6 \%$ and very good particle identification of hadrons in the range of $2-100 \mathrm{GeV}$. A complete description of the $\mathrm{LHCb}$ experiment may be found in Ref. [2].

\section{Measurement of $\sin 2 \beta$}

The process $B^{0} \rightarrow J / \psi K_{\mathrm{S}}^{0}$ is considered as the "golden mode" to measure the CKM $\beta$ angle and was previously used by $B$-factories on $e^{+} e^{-}$ colliders [3].

The final state $J / \psi K_{\mathrm{S}}^{0}$ is common to both the $B^{0}$ and $\bar{B}^{0}$ so CP-violating observables can be defined as:

$$
\begin{aligned}
A(t) & \equiv \frac{\Gamma\left(\bar{B}^{0}(t) \rightarrow J / \psi K_{\mathrm{S}}^{0}\right)-\Gamma\left(B^{0}(t) \rightarrow J / \psi K_{\mathrm{S}}^{0}\right)}{\Gamma\left(\bar{B}^{0}(t) \rightarrow J / \psi K_{\mathrm{S}}^{0}\right)+\Gamma\left(B^{0}(t) \rightarrow J / \psi K_{\mathrm{S}}^{0}\right)} \\
& \simeq S \sin (\Delta m t)-C \cos (\Delta m t) .
\end{aligned}
$$

The parameter $\Delta m$ is the mass difference between the heavy and light mass eigenstates and $S$ and $C$ are CP observables. This decay is sensitive to the phase $\varphi_{d}$ through the interference between the direct decay $B^{0} \rightarrow J / \psi K_{\mathrm{S}}^{0}$ and decay with mixing $B^{0} \rightarrow \bar{B}^{0} \rightarrow J / \psi K_{\mathrm{S}}^{0}$. The phase $\varphi_{d}$ in the frame of the $\mathrm{SM}$ is equal to $\varphi_{d}=2 \beta$, where $\beta$ is defined as: $\beta=\arg \left(-\frac{V_{t d} V_{t b}^{*}}{V_{u d} V_{u b}^{*}}\right)$.

The latest analysis corresponds to an integrated luminosity of $3 \mathrm{fb}^{-1}$ and makes use of a new method of flavour tagging [4]. It is based on more than 41,000 signal events (see the mass distribution in Fig. 3). The fit to the asymmetry function gives: $S=0.731 \pm 0.035$ (stat.) \pm 0.020 (syst.). The $C$ parameter is consistent with zero. These results are consistent with the
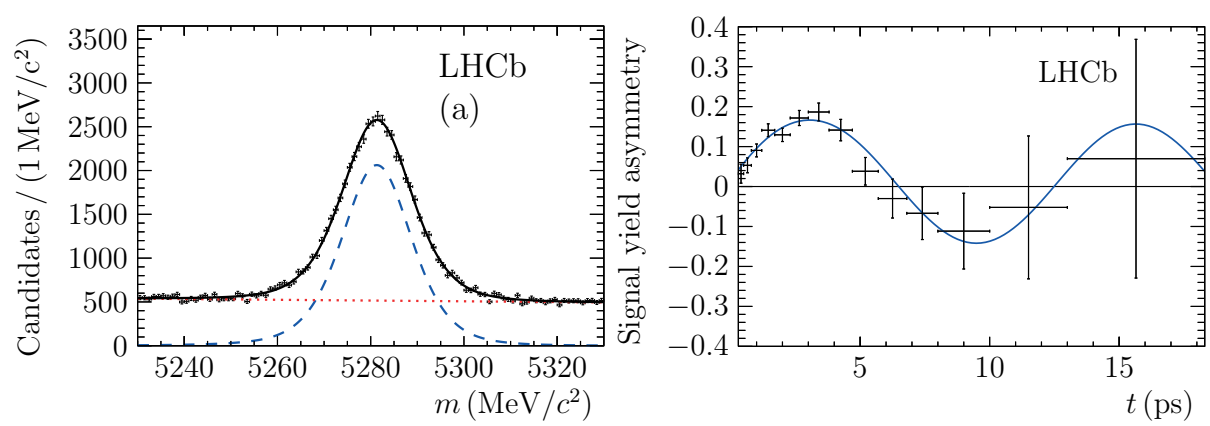

Fig. 3. The mass distribution of $B^{0} \rightarrow J / \psi K_{\mathrm{S}}^{0}$ candidate events (left). Timedependent signal-yield asymmetry of tagged events: $\left(N_{\bar{B}^{0}}-N_{B^{0}}\right) /\left(N_{\bar{B}^{0}}+N_{B^{0}}\right)$ (right). 
current world averages and with expectations from global CKM fits. They are also competitive with $B$-factory results and constitute the most precise time-dependent $\mathrm{CP}$ violation measurement at hadron colliders.

\section{Determination of CKM $\gamma$ angle}

The CKM $\gamma$ angle is still the least accurate measured parameter of the unitary triangle. By contrast, the theoretical predictions are extremely clean since tree-diagram processes only are involved. Its value can help to overconstrain the CKM matrix, constraining the SM as a three generations quark scheme. The $\gamma$ angle is defined as $\gamma \equiv \arg \left(-\frac{V_{u d} V_{u b}^{*}}{V_{c d} V_{c b}^{*}}\right)$ and is, therefore, the phase entering the interference of $b \rightarrow c$ and $b \rightarrow u$ quarks transitions.

The decay $B^{+} \rightarrow D K^{+}$offers various measurement techniques to obtain $\gamma$ angle. The sensitivity of these methods comes from the interference between decays of $D^{0}$ and $\bar{D}^{0}$ to the same final state, as shown on diagram in figure 4 . There are plenty of methods that exploit this phenomenon - their level of importance depends on the relative magnitudes of the suppressed to allowed decay amplitudes of both $B^{+}$and $D^{0}$.

Experimentally, various $D^{0}$ final states may be reconstructed at the LHCb, for example: $D^{0} \rightarrow K^{+} K^{-}, \pi^{+} \pi^{-}, K^{+} K^{-} \pi^{0}, \pi^{+} \pi^{-} \pi^{0}$ (CP eigenstate), $D^{0} \rightarrow K^{+} \pi^{-}, K^{+} \pi^{-} \pi^{+} \pi^{-}, K_{\mathrm{S}}^{0} K^{+} \pi^{-}$(pseudo-flavour specific states) or three-body self-conjugated decays $D^{0} \rightarrow K_{\mathrm{S}}^{0} \pi^{+} \pi^{-}, K_{\mathrm{S}}^{0} K^{+} K^{-}$.

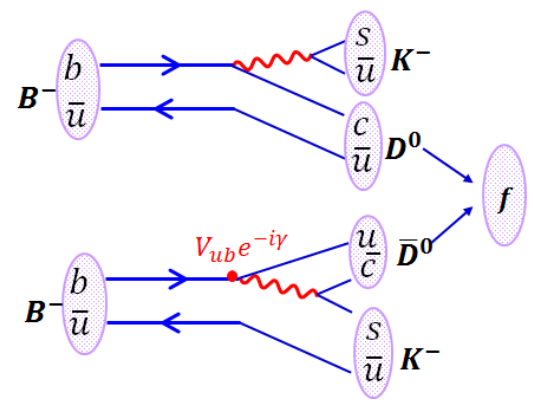

Fig. 4. The diagram showing the $B^{-} \rightarrow D^{0} K^{-}$decay. This is an example process from the rich family of $B \rightarrow D K$ decays, where the sensitivity to the CKM $\gamma$ comes from the interference of $b \rightarrow c$ and $b \rightarrow u$ quark transitions.

For the measurement of the $\gamma$ angle in $B^{+} \rightarrow D^{0}\left(K_{\mathrm{S}}^{00} h^{+} h^{-}\right) K^{+}$, a binned Dalitz analysis is performed [5]. In this method, the sensitivity to the $\gamma$ angle is obtained by comparing the distribution of the events in the $D \rightarrow K_{\mathrm{S}}^{0} h^{+} h^{-}$Dalitz plot for reconstructed $B^{+}$and $B^{-}$mesons. For modelindependent analysis, the knowledge of the strong phase of the $D$ decay over the Dalitz plot is required. 
These measurements lead to the value of $\gamma=\left(62_{-14}^{+15}\right)^{\circ}$ that is the most precise result obtained with a single method.

The so-called ADS and GLW analyses have been performed using $D^{0} \rightarrow$ $K^{+} K^{-} \pi^{0}, \pi^{+} \pi^{-} \pi^{0}$ decays. The relevant mass distributions are shown in Fig. 5. This is the first observation of these channels [6] and it puts new constraint on hadronic parameter $r_{b}$ (ratio of the suppressed to favoured amplitude in $B$ decay): $r_{b}=0.11 \pm 0.03$.

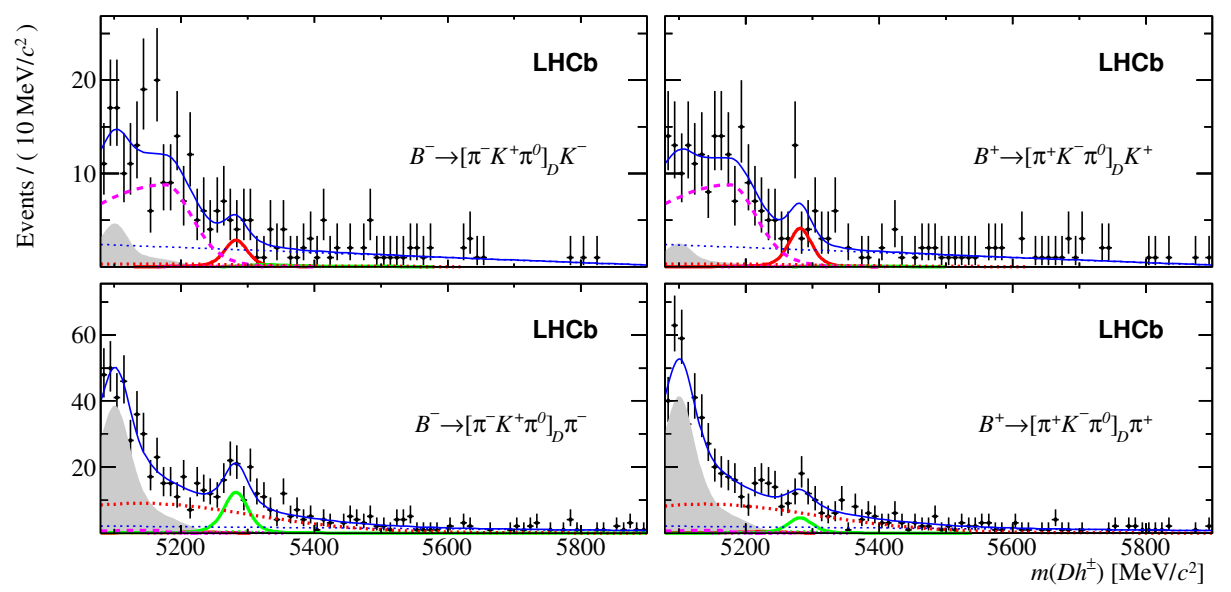

Fig. 5. The invariant mass distribution of the system $\pi^{-} K^{+} \pi^{0}$ in the $\operatorname{ADS} B^{+} \rightarrow$ $D K^{+}$analysis (top) and $B^{+} \rightarrow D \pi^{+}$(bottom) separated by the respective $B$ meson charge (left and right).

The $B^{-}$reconstruction through the inclusive ADS mode $B^{-} \rightarrow D X_{s}^{-}$, where $X_{s}^{-}=K^{-} \pi^{+} \pi^{-}$, and $D \rightarrow h^{+} h^{-}$offers a new constraint on CKM $\gamma$ angle. Although much better sensitivity is expected in suppressed modes (see Fig. 6), the huge statistics of LHCb data leads to the result: $\gamma=$ $\left(74_{-19}^{+20}\right)^{\circ}[7]$.

The CKM $\gamma$ angle can be also extracted using the time-dependent study of the $B_{s}^{0} \rightarrow D_{s}^{ \pm} K^{\mp}$ decay. The time-dependent decay rate depends on $\sin \left(\gamma+2 \beta_{s}\right)$ and strong phases. Since one needs to measure four time-dependent decay rates and use flavour tagging, it is experimentally challenging.

The mass distribution, the $B_{s}^{0}$ decay time and asymmetry of tagged events are presented in Fig. 7. The first measurement performed with this method yields the result: $\gamma=\left(115_{-43}^{+28}\right)^{\circ}$ (see also the 1-CL profile in Fig. 7) using the data sample that corresponds to an integrated luminosity of $1 \mathrm{fb}^{-1}$ [8]. To achieve the ultimate precision on $\gamma$, this very sophisticated method requires not only the whole Run 1 sample but also the data to be collected during Run 2 and beyond. 

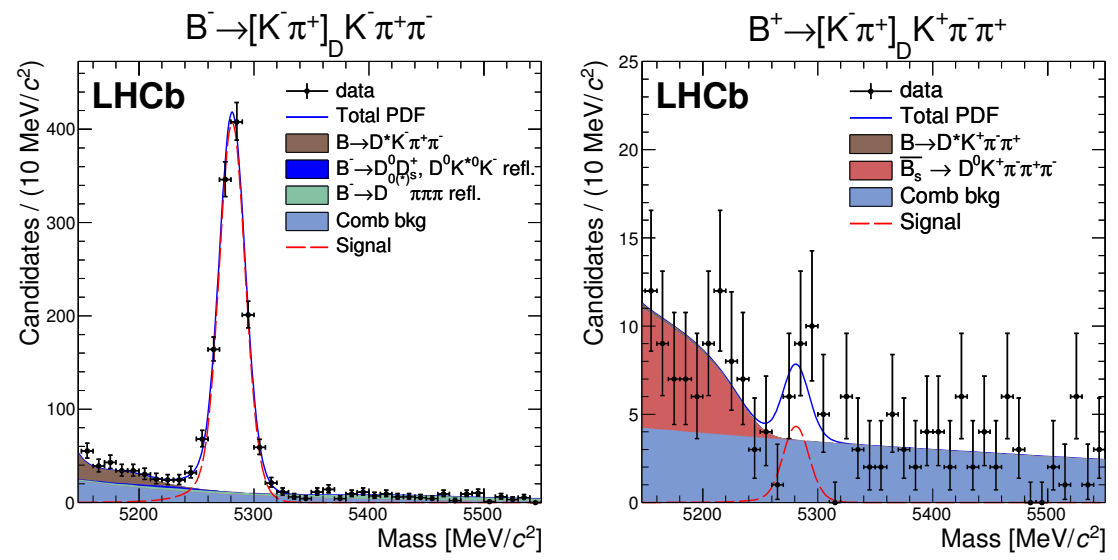

Fig. 6. The invariant mass distribution of the reconstructed system $B^{-} \rightarrow D X_{s}^{-}$using ADS selection. The decay $B^{-} \rightarrow\left[K^{-} \pi^{+}\right]_{D} K^{-} \pi^{+} \pi^{-}$(left) is favoured, whereas the $B^{+} \rightarrow\left[K^{-} \pi^{+}\right]_{D} K^{+} \pi^{-} \pi^{+}$(right) is doubly suppressed.
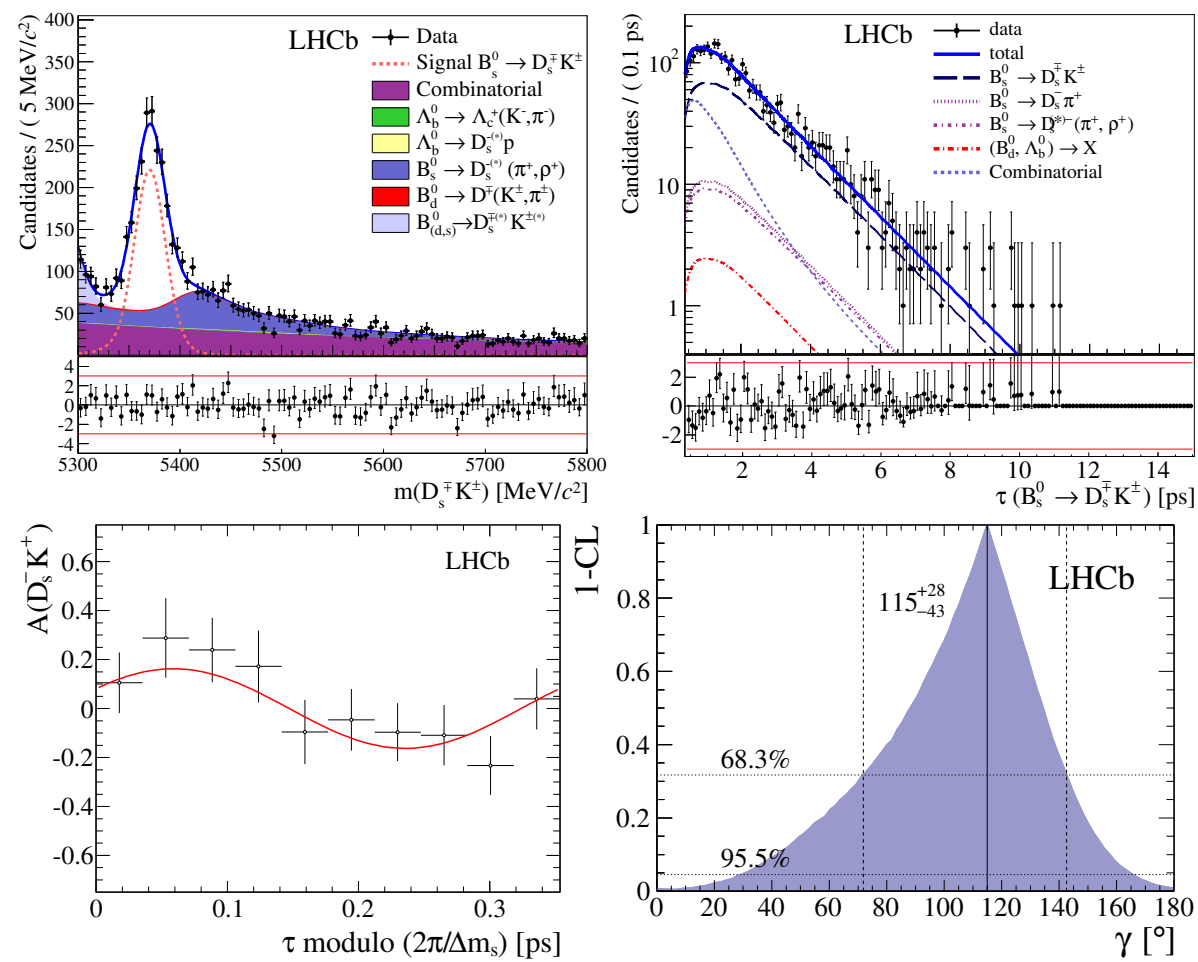

Fig. 7. The invariant mass distribution of the system $B_{s}^{0} \rightarrow D_{s} K$ (top left); decay time of $B_{s}^{0}$ candidates (top right); the asymmetry in the rates of tagged $B_{s}^{0}$ and $\bar{B}_{s}^{0}$ decaying to $D_{s}^{-} K^{+}$(bottom left); and the 1-CL profile for the $\gamma$ angle (bottom right). 
A new combination of all $\gamma$ measurements performed by LHCb has been recently made [9]. The combination of all results gives the best fit value of $\gamma=\left(70.9_{-8.5}^{+7.1}\right)^{\circ}$ at $68 \%$ C.L. This is the most precise single-experiment measurement of the CKM angle $\gamma$ to date.

\section{Direct $\mathrm{CP}$ violation in $B$ decays}

Direct $\mathrm{CP}$ violation (also called $\mathrm{CP}$ violation in decays) occurs when two amplitudes with different weak and strong phases interfere. In this type of analysis, one looks for the difference in the decay rates of charge conjugate decays. In the case of neutral $B$ meson decays, the asymmetry is given by $A_{\mathrm{CP}}\left(B_{(s)}^{0} \rightarrow f_{(s)}\right)=\frac{\Gamma\left(\bar{B}_{(s)}^{0} \rightarrow \bar{f}_{(s)}\right)-\Gamma\left(B_{(s)}^{0} \rightarrow f_{(s)}\right)}{\Gamma\left(\bar{B}_{(s)}^{0} \rightarrow \bar{f}_{(s)}\right)+\Gamma\left(B_{(s)}^{0} \rightarrow f_{(s)}\right)}$. In the case of charged $B$ meson decays $B^{ \pm} \rightarrow h^{ \pm} h^{+} h^{-}$, very different asymmetries may be observed in various regions of the phase space.

The $K^{ \pm} \pi^{\mp}$ mass distribution is presented in Fig. 8. The simultaneous fit to the experimental mass spectrum (containing both $B^{0}$ and $B_{s}^{0}$ mesons) shows a clear asymmetry between the charge conjugated final states.

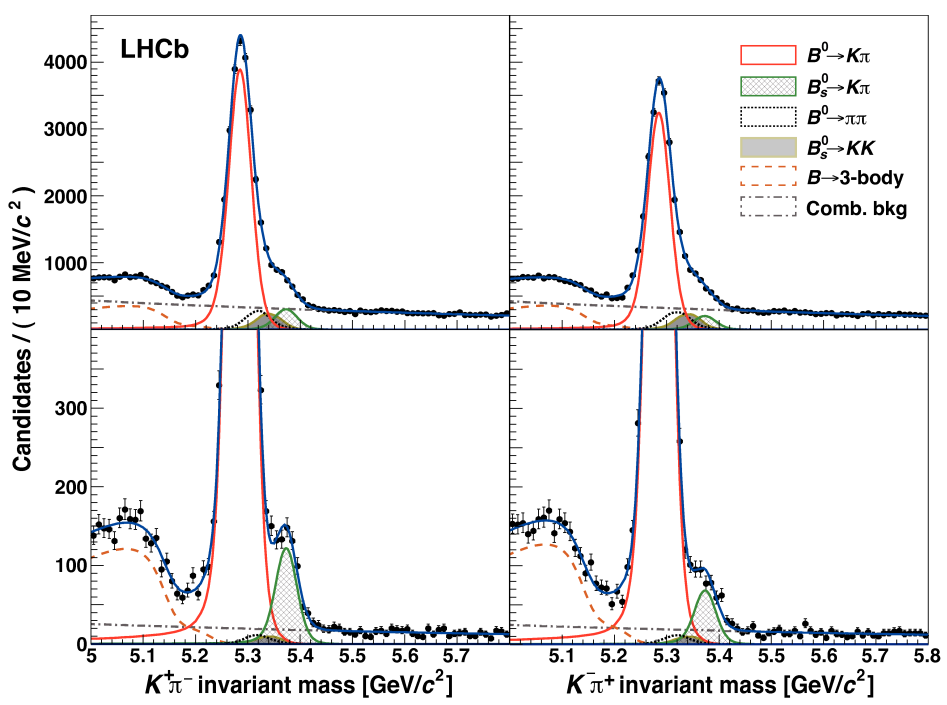

Fig. 8. The mass distribution of the $K^{+} \pi^{-}$(left) and $K^{-} \pi^{+}$(right) of the selected $B_{(s)}^{0} \rightarrow K \pi$ events.

The CP asymmetries are derived from raw signal yields, taking into account effects induced by the detector acceptance, event reconstruction, other asymmetries caused by the detector and production dynamics. The 
$\mathrm{CP}$ asymmetries that have been measured are [10]:

$$
\begin{aligned}
& A_{\mathrm{CP}}\left(B^{0} \rightarrow K^{+} \pi^{-}\right)=-0.080 \pm 0.007 \pm 0.003, \\
& A_{\mathrm{CP}}\left(B_{s}^{0} \rightarrow K^{+} \pi^{-}\right)=027 \pm 0.04 \pm 0.001 .
\end{aligned}
$$

The former is the most precise measurement to date, whereas the latter is the first observation of direct CP violation in $B_{s}^{0}$ meson decays.

The charmless three-body decays $B^{ \pm} \rightarrow h^{ \pm} h^{+} h^{-}$, where $h$ stands for charged kaon or pion, enable the study of different sources of CP violation through interference of various resonances in different regions in Dalitz plot. For all final states, the same selection criteria are used and the signal yields are obtained. The invariant mass spectra of two decay modes are presented in Fig. 9. Fits are made separately for each data sample taking into account the specific detection and trigger conditions. In these analyses, corrections for production and detection asymmetries are also implemented.

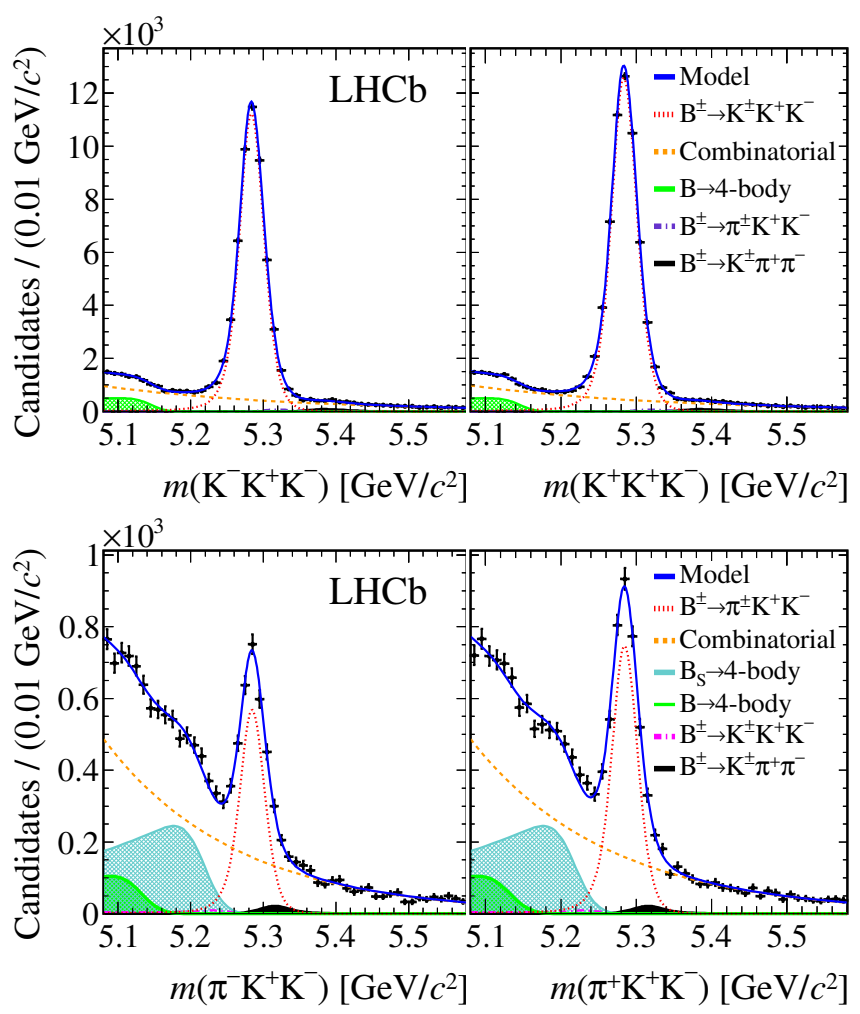

Fig. 9. The mass distribution of the three hadron (charged kaons or pions) final states. The left panels show the $B^{-}$candidates and the right ones the $B^{+}$candidates. 
The results obtained for the integrated CP asymmetries are [11]:

$$
\begin{aligned}
A_{\mathrm{CP}}\left(B^{ \pm} \rightarrow K^{ \pm} \pi^{+} \pi^{-}\right) & =+0.025 \pm 0.004 \pm 0.004, \\
A_{\mathrm{CP}}\left(B^{ \pm} \rightarrow K^{ \pm} K^{+} K^{-}\right) & =-0.036 \pm 0.004 \pm 0.002, \\
A_{\mathrm{CP}}\left(B^{ \pm} \rightarrow \pi^{ \pm} \pi^{+} \pi^{-}\right) & =+0.058 \pm 0.008 \pm 0.009 \\
A_{\mathrm{CP}}\left(B^{ \pm} \rightarrow \pi^{ \pm} K^{+} K^{-}\right) & =-0.123 \pm 0.017 \pm 0.012 .
\end{aligned}
$$

The rich spectrum of resonances created in these three-body final states opens the possibility for studying effects of direct $\mathrm{CP}$ violation in different regions of phase space. Figure 10 shows the raw charge asymmetry of event yields in different bins of the Dalitz plot. These distributions reveal various sources of CP violation such as $\pi^{+} \pi^{-}$and $K^{+} K^{-}$rescattering or interference between the $\mathrm{S}$ - and $\mathrm{P}$-wave around $\rho(770)$ mass. For a detailed discussion of these very interesting and promising effects, see [11].
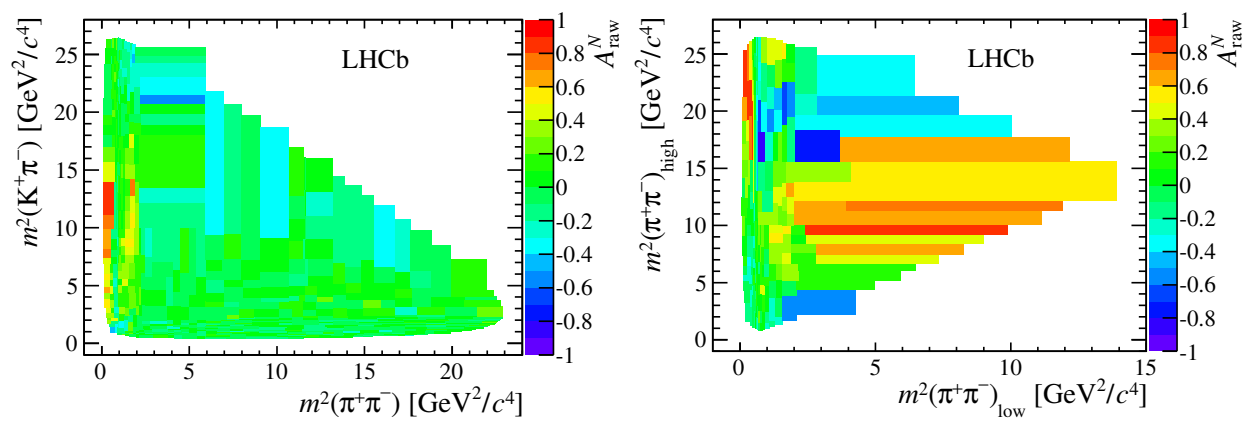

Fig. 10. The charge asymmetry in Dalitz plot bins $B^{ \pm} \rightarrow K^{ \pm} K^{+} K^{-}$(left) and $B^{ \pm} \rightarrow \pi^{ \pm} \pi^{+} \pi^{-}$(right) decays.

\section{Search for CP violation in charm}

$\mathrm{CP}$ violation in the charm sector is expected to be tiny in the $\mathrm{SM}$ - at the level equal or smaller than $10^{-3}$. On the other hand, any enhancement would be a clear signal of new physics effects.

The CP asymmetry has been measured using the $D^{0}$ decay rates to $\mathrm{CP}$ eigenstates $D^{0} \rightarrow K^{+} K^{-}$and $D^{0} \rightarrow \pi^{+} \pi^{-}$, where $D^{0}$ comes from the decays $B^{0} \rightarrow D^{0} \mu^{-} X$ and the $D^{0}$ flavour is tagged by the sign of the muon.

The mass distribution of the $K^{+} K^{-}$system and the corresponding asymmetry are shown in Fig. 11. 

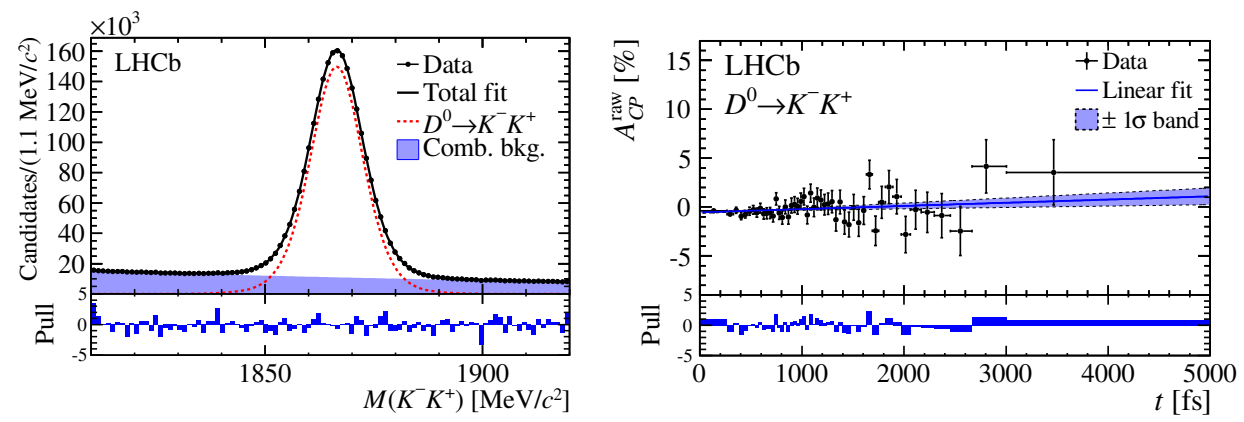

Fig. 11. The invariant mass distribution for $D^{0} \rightarrow K^{+} K^{-}$(left). The raw CP asymmetry as a function of $D^{0}$ decay time for $D^{0} \rightarrow K^{+} K^{-}$(right).

$\mathrm{CP}$ asymmetry in the charm sector includes both indirect and direct contributions. The values for the observable $A_{\Gamma}$ obtained in the latest analysis are [12]:

$$
\begin{aligned}
A_{\Gamma}\left(K^{+} K^{-}\right) & =-0.134 \pm 0.077_{-00034}^{+0.026} \%, \\
A_{\Gamma}\left(\pi^{+} \pi^{-}\right) & =-0.092 \pm 0.145_{-00033}^{+0.025} \% .
\end{aligned}
$$

No evidence of discrepancies from the SM has been found so far.

\section{Summary and prospect for new measurements in Run 2}

The LHCb spectrometer features outstanding tracking and vertexing capabilities and has already demonstrated high ability to perform precise measurements in the heavy flavour sector. The $\sin 2 \beta$ parameter was measured by $\mathrm{LHCb}$ with a precision comparable to that from the $B$-factories. The CKM $\gamma$ angle was determined with the best precision to date and with new methods. Direct CP violation has been observed in neutral and charged $B$ meson decays. Still, there are no significant indications of any new physics phenomena neither in beauty nor in charm to date.

The energy of colliding protons in LHC was raised to $\sqrt{s}=13 \mathrm{TeV}$ and a new period (Run 2) started in 2015. Significant improvements have been implemented in the software of the High Level Trigger. The main objective is to achieve the off-line quality tracking in real-time conditions. That requires that the detector alignment and calibration (crucial for physics performance) have to be also performed in (quasi) real-time on a fill-by-fill basis. Almost identical performance of the on-line and off-line reconstruction makes sure that the same candidates are taken in both stages [13].

First data taken in 2015 showed that owing to the fact that the $b \bar{b}$ cross section is about two times larger than in Run 1 and that the bunch spacing is reduced, thus lowering the occupancy per crossing, the LHCb managed 
to improve its physics performance. In addition, a novel way of raw data processing (so-called Turbo stream) has been implemented. The basic idea is simple - in this special stream, only objects (tracks and vertices) that pass the specified criteria are saved, reducing the size of stored data and thus increasing the event rate. Benefiting from this method, the LHCb managed to obtain excellent results in the charm sector even with a very small data sample taken during year 2015 [14].

The new approach to the on-line event selection has a special meaning for obtaining the better precision on the $\gamma$ angle, since the current experimental uncertainties are large. More data, in particular in suppressed and multibody modes, are needed. The time-dependent analysis needs not only more signal, tagged events, but also new decay channels (for example, $B_{s}^{0}$ $\left.B_{s}^{0} \rightarrow D_{s}^{* \pm} K^{* \mp}\right)[15]$. During Run 2 data corresponding to $10 \mathrm{fb}^{-1}$ of integrated luminosity are expected to be accumulated and the precision on the angle $\gamma$ should reach $\sigma(\gamma) \sim 4^{\circ}$.

\section{REFERENCES}

[1] http://ckmfitter.in2p3.fr/www/html/ckm_results.html

[2] A.A. Alves Jr. et al. [LHCb Collaboration], JINST 3, S08005 (2008).

[3] B. Aubert et al. [BaBar Collaboration], Phys. Rev. Lett. 87, 091801 (2001). K. Abe et al. [Belle Collaboration], Phys. Rev. Lett. 87, 091802 (2001).

[4] R. Aaij et al. [LHCb Collaboration], Phys. Rev. Lett. 115, 031601 (2015).

[5] R. Aaij et al. [LHCb Collaboration], J. High Energy Phys. 1410, 097 (2014).

[6] R. Aaij et al. [LHCb Collaboration], Phys. Rev. D 91, 112014 (2015).

[7] R. Aaij et al. [LHCb Collaboration], Phys. Rev. D 92, 112005 (2015).

[8] R. Aaij et al. [LHCb Collaboration], J. High Energy Phys. 1411, 060 (2014).

[9] R. Aaij et al. [LHCb Collaboration], Measurement of the CKM Angle $\gamma$ from a Combination of $B \rightarrow D K$ Analyses, LHCb-CONF-2016-001.

[10] R. Aaij et al. [LHCb Collaboration], Phys. Rev. Lett. 110, 221601 (2013).

[11] R. Aaij et al. [LHCb Collaboration], Phys. Rev. D 90, 112004 (2014).

[12] R. Aaij et al. [LHCb Collaboration], J. High Energy Phys. 1504, 043 (2015).

[13] G. Dujany, B. Storaci, J. Phys.: Conf. Ser. 664, 082010 (2015).

[14] R. Aaij et al. [LHCb Collaboration], J. High Energy Phys. 1603, 159 (2016) [arXiv:1510.01707 [hep-ex]]; 1510, 172 (2015) [arXiv:1509.00771 [hep-ex]].

[15] R. Aaij et al. [LHCb Collaboration], J. High Energy Phys. 1506, 130 (2015). 